\section{Behavior of Hydroxycinnamates, Related Aldehydes and Alcohols in Rice Leaves Infected by Blast Fungus with Reference to Inducible Lignification ${ }^{\dagger}$}

\author{
Minoru Haga, Hisato Ogata, \\ Yasuharu Sekizawa ${ }^{\dagger \dagger}$ Seishi Shibahara* \\ and Hiroshi KuriHara* \\ Faculty of Agriculture, Tamagawa University, \\ Machida-shi, Tokyo 194, Japan \\ * Research Laboratories, Meiji Seika Kaisha Ltd., \\ Kohoku-ku, Yokohama 222, Japan
}

Received September 10, 1990

As previously reported, ${ }^{1)}$ the lignification in rice leaves was markedly induced by infection with blast fungus, and the spectrophotometric characteristics of dioxane lignins separated from either healthy or diseased leaves were clearly different, indicating that the contents of threc precursors were unalike within each lignin. In this paper, the dynamic behavior of free or bound hydroxycinnamic acids, hydroxycinnamic aldehydes and alcohols, both in healthy and diseased rice leaves, was determined by high-performance liquid chromatogtaphy (HPLC), using a ZORBAX ODS $(4.6 \times 250 \mathrm{~mm})$ column with $1 \%$ acetic acid in methanol-water $(20: 80, v / v)$ and scanning at $254 \mathrm{~nm}$. The rice cultivar and blast fungus race used were a similar combination to that previously reported. ${ }^{1)}$ The experimental conditions of spray inoculation of the conidia and maintaining the inoculated and non-inoculated rice seedlings were also similar as those in the provious report. ${ }^{1)}$ As 4-Coumaryl (C. alc.), coniferyl (Cf. alc.) and sinapyl alcohol (Sn. alc) or aldehydes (C. ald., Cf. ald. and Sn. ald.) were not available from commercial sources, they were independently prepared. Ten m moles of 4-coumaric, ferulic or sinapic acid was dissolved in $150 \mathrm{ml}$ of tetrahydrofuran. To the solution, $40 \mathrm{mg}$ of lithium aluminum hydride was added, and the mixture was stirred for $3 \mathrm{hr}$ at room temperature. After filtration, the filtrate was concentrated, and the resultant concentrate was subjected to Kieselgel (Merck 60F254) thin-layer chromatography, using a developing solvent of hexane-ethyl acetate $(1: 1)$. A band of alcohol was identified by UV detection, scraped off and eluted by ethyl acetate. After concentration to dryness, the product was identified by IR, mass and NMR spectroscopic method. Each of $\mathrm{C}$. alc., Cf. alc. and $\mathrm{Sn}$. alc. (3.3 $\mathrm{mm}$ each) was dissolved in $20 \mathrm{ml}$ of tetrahydrofuran. To each solution, $5 \mathrm{~m}$ moles of pyridinium chlorochromate was added, and the mixture was stirred for 1 hr at room temperature. After filtration, the filtrate was concentrated and the resultant concentrate was applied to Kieselgel (Merck 60F254) thin-layer chromatography, using a developing slovent of hex-

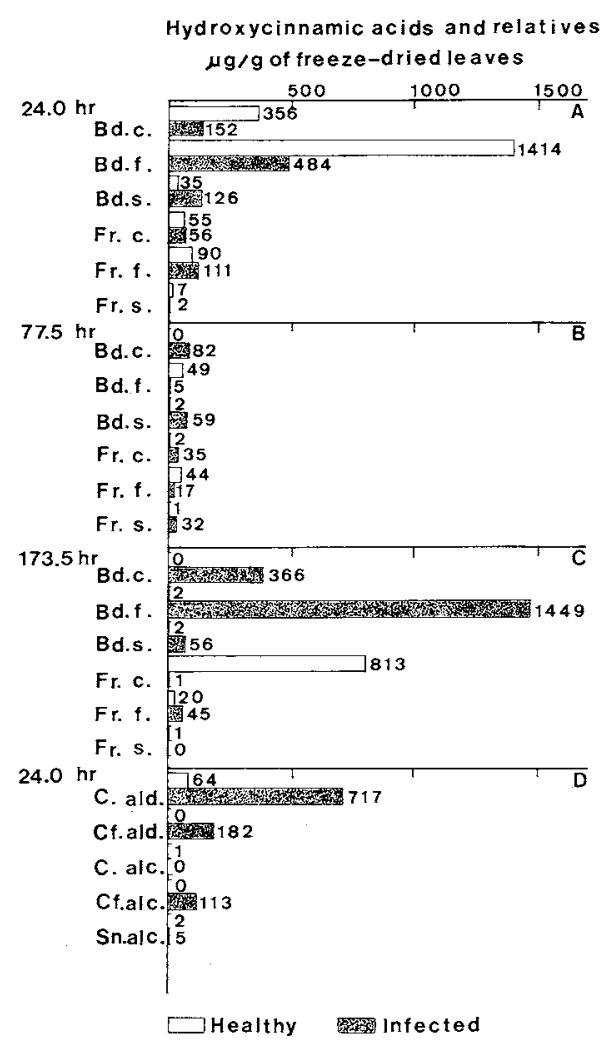

Fig. 1. Dynamic Behavior of Hydroxycinnamic Acids, Their Aldehydes and Alcohols in either Healthy or Infected Rice Blades.

Bd. c., bound 4-coumaric acid; Bd. f., bound ferulic acid; Bd. s., bound sinapic acid; Fr. c., free 4-coumaric acid; Fr. f., free ferulic acid; Fr. s., free sinapic acid; C. ald., 4-coumaraldehyde; Cf. ald., coniferaldehyde; C. alc., 4-coumaryl alcohol; Cf. alc., coniferyl alcohol; Sn. alc., synapyl alcohol. Sinapaldehyde (Sn. ald.) was not detected throughout the experiments.

+ Causality Analysis on Cascades of Reactions in the Induced Defense Mechanism of Rice plant. Part XI. This work was supported in part by a Grant-in-Aid for Scientific Research (No. 01480055) from the Ministry of Education, Science and Culture of Japan.

t† To whom inquiries and requests for reprints should be addressed. 
ane-ethyl acetate (2:1). A band of aldehyde was identified by UV detection, scraped off and eluted by ethyl acetate. After concentrating to dryness, the product was identified by IR, mass- and NMR spectroscopic method. Rice leaves, either from healthy or diseased rice seedlings, were freeze dried and stored at $-20^{\circ} \mathrm{C}$. After pulverizing of the dried leaves, separation of the free or bound hydroxycinnamic acids, their aldehydes and alcohols was done according to Sosulski et $a l^{2)}$ and Andersen et al. ${ }^{3)}$ The results from quantitative analyses by HPLC are summarized in Fig. 1. It was noted that an extensive decrease of both bound ferulic (Bd. f.) and 4-coumaric acid (Bd. c.) occurred in the infected rice leaves after the inoculation procedure in a saturated moist-air chamber at $25^{\circ} \mathrm{C}$ (Fig. 1A). This suggested that the activation or induction of an esterase to hydrolyze the bound hydroxycinnamates in the cell wall ${ }^{4)}$ was operable at a relatively early stage of the infection. However, free ferulic (Fr. f.) and 4-coumaric acid (Fr. c.) had not accumulated at this stage (Fig. 1A), suggesting that both the acids were further reduced to their aldehydes along the biosynthetic pathway for lignin. The temporary accumulation of 4-coumaraldehyde (C. ald.) in the infected leaves at the relatively early stage of infection seems likely to confirm this hypothesis (Fig. ID). The accummulation of coniferaldehyde (Cf. ald.) was less (Fig. ID), suggesting that the coniferaledyde might be further reduced to coniferyl alcohol, which could be utilized as a major precursor to form lignin in the infected rice leaves. This seemes consistent with the IR-spectrophotometric properties of the lignin separated from the diseased rice leaves that were previously reported. ${ }^{11}$ In this experimental system, free and bound sinapic acid, the aldehyde and alcohol did not show any appreciable behavior. The bound and free hydroxycinnamic acids, their aldehydes and alcohols in both the healthy and infected leaves had decreased at $77.5 \mathrm{hr}$ after the inoculation (Fig. 1B). This suggests that hydroxycinnamic acids in the healthyl leaves were also mobilized for the lignification of young 5 th rice leaves that had just emerged. It was further noted that the contents of both bound ferulic (Bd. f.) and 4-coumaric acid (Bd. c.) in the infected leaves at $173.5 \mathrm{hr}$ after the inoculation had been restored to level existing in the healthyl leaves $24 \mathrm{hr}$ after inoculation (Figs. IC and 1A), while these levels in the healthyl leaves did not regain the initial level, because there was a marked accumulation of free 4-coumaric acid (Figs. 1C and 1A). The content of aldehydes and alcohols was almost exhausted in the period from 77.5 to $173.5 \mathrm{hr}$ after the inoculation (data not shown). Bound diferulic acid ${ }^{53}$ was not detected in either the healthy or diseased rice leaves. This study revealed that (1) ferulate and 4-coumarate bound to the cell wall were hydrolized at the the early stage of blast fungus infection to feed precursors for lignin biosynthesis; (2) the dual feeding systems of hydroxycinnamic acids for lignin biosynthesis were operable in the host plant, one being initiated by the induction of phenylalanine ammonia-lyase in the infected host plant, ${ }^{6)}$ and the other by the hydrolytic release of hydroxycinnamic acids bound to the cell walls; and (3) the major hydroxycinnamic acid utilized for lignin biosynthesis was ferulic acid. A comparative study between compatible and incompatibie combinations remains to be done.

Acknowledgments. The authors are grateful to Dr. N. Shibuya of the National Food Research Institute at Tsukuba for his generous gift of authentic diferulic acid. Thanks are also given to Mrs. T. Yasukawa for her assistance in preparing this manuscript.

\section{References}

1) M. Haga, H. Ogata and Y. Sekizawa, Agric. Biol. Chem., 55, 619 (1991).

2) F. Sosulski, K. Krygier and L. Hogge, J. Agric. Food Chem., 30, 337 (1982).

3) R. A. Andersen, T. H. Vaughn and M. J. Kasperbauer, J. Agric. Food Chem., 28, 427 (1980).

4) S. C. Fry, Biochem. J., 203, 493 (1982).

5) H. U. Markwalder and H. Neukom, Phytochemistry, 15, 836 (1976).

6) M. Haga, T. Haruyama, H. Kanoh, Y. Sekizawa, S Urushizaki and K. Matsumoto, Agric. Biol. Chem., 52, 943 (1988). 